\title{
Measurement of neutron spectra for photonuclear reaction with linearly polarized photons
}

\author{
Yoichi Kirihara ${ }^{1, *}$, Toshiro Itoga ${ }^{2}$, Toshiya Sanami ${ }^{1,4,5}$, Hiroshi Nakashima $^{3,4}$, Yoshihito Namito ${ }^{1,5}$, Shuji Miyamoto ${ }^{6}$, \\ Akinori Takemoto ${ }^{6}$, Masashi Yamaguchi $^{6}$, and Yoshihiro Asano ${ }^{4,6}$ \\ ${ }^{1}$ High Energy Accelerator Research Organization, 1-1 Oho, Tsukuba, Ibaraki 305-0801, Japan \\ ${ }^{2}$ JASRI, 1-1-1 Kouto, Sayo-cho, Sayo-gun, Hyogo 679-5198, Japan \\ ${ }^{3}$ Japan Atomic Energy Agency, 2-4 Shirakata, Tokai-mura, Naka-gun, Ibaraki 319-1195, Japan \\ ${ }^{4}$ RIKEN SPring-8 Center, 1-1-1 Kouto, Sayo-cho, Sayo-gun, Hyogo 679-5198, Japan \\ ${ }^{5}$ The Graduate University for Advanced Studies (SOKENDAI), 1-1 Oho, Tsukuba, Ibaraki 305-0801, Japan \\ ${ }^{6}$ University of Hyogo, 1-1-2, Koto, Kamigori-cho, Ako-gun, Hyogo 678-1205, Japan
}

\begin{abstract}
Spectra of neutrons produced by a photonuclear reaction from a ${ }^{197} \mathrm{Au}$ target were measured using $16.95 \mathrm{MeV}$ linearly and circularly polarized photon beams at NewSUBARU-BL01 using a time-offlight method. The difference in the neutron spectra between the cases of a linearly and circularly polarized photon was measured. The difference in the neutron yield increased with the neutron energy and was approximately threefold at the maximum neutron energy. In a direction perpendicular to that of the linear polarization, the neutron yields decreased as the neutron energy increased.
\end{abstract}

\section{Introduction}

In high-energy electron-accelerator facilities, electrons hitting components of the accelerators produce highenergy photons through bremsstrahlung. The photons generate fast neutrons through photo-nuclear reactions. The neutrons induce issues in radiation protection, for example, increase of ambient dose behind a shielding wall and production of activity in air and surrounding materials. To evaluate these issues, the data on not only neutron production but also neutron energy and angular distribution are important for photo-nuclear reactions.

For instance at the International Linear Collider (ILC) facility, polarized photons with hundreds of $\mathrm{kW}$ impinge on a target to produce positrons [1]. Thus, from the target, significant amount of neutrons are produced as a result of photo-nuclear reactions. The neutron production form the reaction should be estimated reasonably well for radiation shielding design of the ILC.

Experimental data for neutron production of photonuclear reaction, until now, have been taken intensively by Livermore and Saclay groups for giant dipole resonance (GDR) region [2]. Recently, the anisotropy of neutron azimuthal angular distributions with linearly polarized photon beams was measured by Horikawa et al. in GDR region [3].

Nevertheless, the experimental data on neutron energy and angular distribution are still not sufficient since most of the data were obtained with low intensity photon sources. Furthermore, effects on neutron energy distribution due to polarization are not obvious since Horikawa et al. provided data based on relative neutron yield [3]. They didn't take data for un-polarized photon to reveal enhancement on neutron production due to polarization.

On the other hand, reaction model of photo nuclear reaction was proposed by Agodi [4]. Thee consequence of the model was partially confirmed by the data of Horikawa et al. [3]. This model, however, has not been included in the particle-transport codes that are used for shielding design of high-energy accelerators. Thus, to develop and evaluate a reaction model to treat the effect of polarization in particle transport codes, experimental data are desired on neutron energy and angular distribution for linear and circular polarization photons.

Recently, intense mono-energy photon beam up to 70 $\mathrm{MeV}$ become available at NewSUBARU-BL01. University of Hyogo, Japan, using laser Compton scattering (LCS) technique $[5,6]$. The polarization of LCS photon can be easily controlled by changing polarization of laser system. The usage of the LCS photon enables us to take systematic data of neutron energy and angular distribution for mono-energetic photons with considering effects from polarization.

In this study, as a part of the systematic data taking, we obtain the data of neutron energy and angular dependency to reveal effect of polarization. We use not only linearly polarized photon beam but also circularly polarized to obtain difference between them. To obtain absolute neutron spectra for the photons, incident number of photons and neutron detector efficiency are carefully determined experimentally, which are not considered in ref. 2. As a material of the neutron production target, ${ }^{197} \mathrm{Au}$ was chosen since it showed large polarization dependence [3].

* Corresponding author: kyoichi@post.kek.jp 


\section{Experimental}

\subsection{Experimental set up}

An overview of BL01 and how to obtained LCS photons are described elsewhere [3, 5], only outline is summarized in this section.

Figure 1 shows schematic drawing of BL01. An LCS photon beam was generated by scattering laser photons and electron bunches in a storage ring. The storage ring was operated in the single bunch with top-up mode. The typical stored beam current was $30 \mathrm{~mA}$ during the experiment. The electron energy was $982.4 \mathrm{MeV}$. An $\mathrm{NdYVO}_{4}$ laser system provided laser photons with a wavelength for the laser photon of $1.064 \mu \mathrm{m}$. The laser power was $20 \mathrm{~W}$. Under these conditions, the maximum energy of the LCS photon beam was $16.95 \mathrm{MeV}$ at a scattering angle of $180^{\circ}$. The LCS photon beam was collimated using a 3-mm-inner-diameter collimator in an enclosure of the storage ring, $13.82 \mathrm{~m}$ away from the collision point, and a 2-mm-inner-diameter collimator in optics hutch 1, $16.67 \mathrm{~m}$ from the collision point. After passing through these collimators, the beam is led to an optics hutch 2.

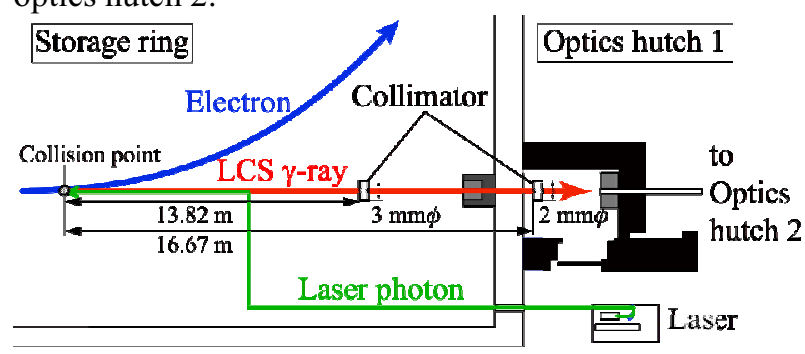

Fig. 1. Schematic of NewSUBARU-BL01.

Figure 2 shows the experimental setup in the optics hutch 2 . At the entrance of the hutch, a plastic scintillator plate with thickness of $5 \mathrm{~mm}$ coupled with photomultiplier tube was placed on the path of LCS photon beam to monitor relative intensity of the beam (PLS in Figure 2). $1.4 \mathrm{~m}$ downstream from the PLS, a cylindrical shaped ${ }^{197} \mathrm{Au}$ target was placed. The axis of the target was parallel to the beam line. The diameter and thickness of the target were 10 and $40 \mathrm{~mm}$, respectively. Although the neutron attenuates within the target, this attenuation was not considered in this paper.

We used three NE213 organic liquid scintillators, $12.7 \mathrm{~cm}$ in diameter and $12.7 \mathrm{~cm}$ in thickness, coupled with photo-multiplier tubes as neutron detectors. The positions of the NE213 detectors are shown in Figure 2 and listed in Table 1. The pulse height NE213 detectors were calibrated using $\gamma$-ray sources prior to the beam experiment. The detection efficiencies for each detector were determined as a function of the pulse height bias (see section 3.3 in detail).

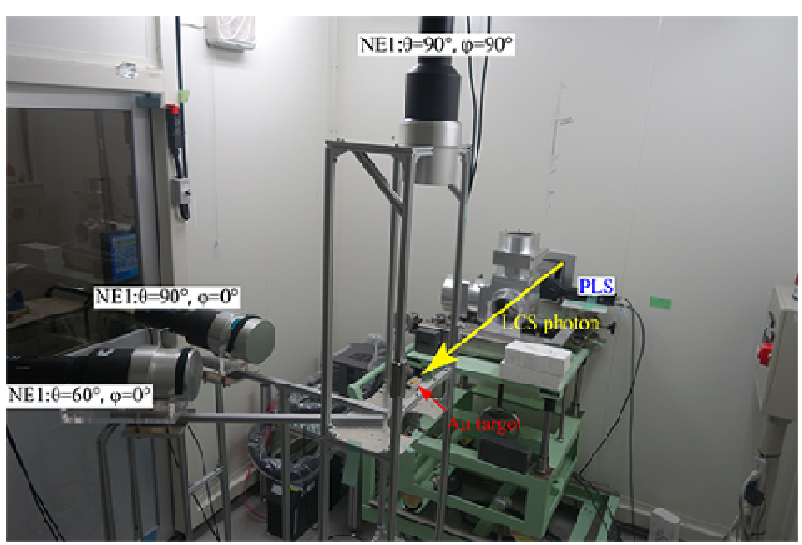

Fig. 2. Experimental setup used in this study.

Table 1. Positions of the NE213 detectors.

\begin{tabular}{|c|c|c|c|}
\hline $\begin{array}{c}\text { Detector } \\
\text { label }\end{array}$ & $\begin{array}{c}\text { Flight path } \\
\text { from target } \\
{[\mathrm{cm}]}\end{array}$ & $\begin{array}{c}\text { Polar angle } \\
\theta \text { [degrees] }\end{array}$ & $\begin{array}{c}\text { Azimuthal } \\
\text { angle } \phi \\
\text { [degrees] }\end{array}$ \\
\hline NE1 & 70.9 & 60 & 0 \\
\hline NE2 & 64.7 & 90 & 0 \\
\hline NE3 & 65.9 & 90 & 90 \\
\hline
\end{tabular}

\subsection{Readout electronics}

Figure 3 shows a block diagram of the readout electronics. Analogue signals from NE213 detectors were divided to three to obtain pulse integral data $(\mathrm{PH})$ with total and slow gates for pulse shape discrimination (PSD) and time difference from RF signal for time-offlight (TOF) measurement. Only beam coincident events were selected using 4 fold 1 veto coincidence module to generate the gate and TDC start signal.

The RF signal was also used to trigger laser to generate LCS photon [7] (not shown in this figure) and TDC stop signal.

The analogue signal from PLS was divided to two signals to obtain total pulse integral.

The data acquisition system is $\mathrm{A} 3000$ system that consists of QDC of A3200, TDC of A3300 modules and VME controller (Nikiglass Co.Ltd.). The modules were operated free-run list mode that accumulates data from these modules with adding time stamp with $\mathrm{XX}$ ns resolution event by event. The time stamp was used to find out a pair of PH and TOF data in offline analysys. The data were stored in HDD of Linux PC that was connected with VME controller via gigabit Ethernet interface. 


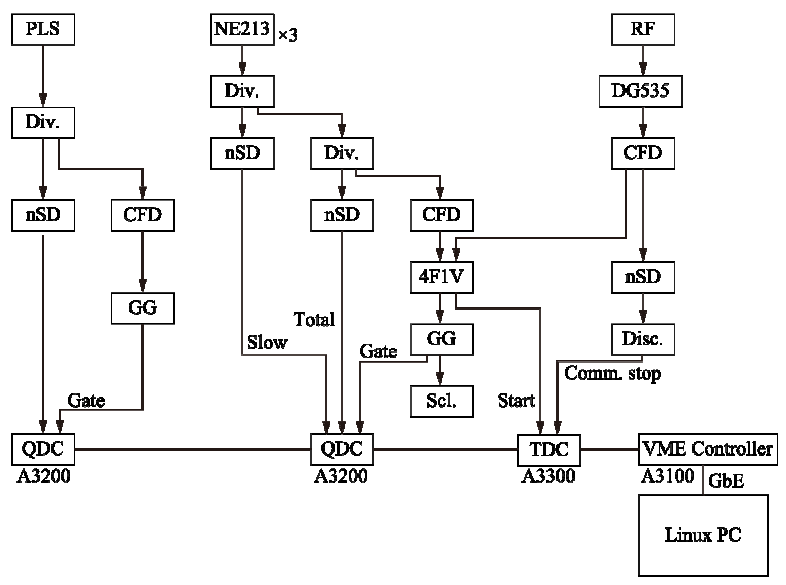

Fig. 3. Block diagram of the electronics circuit. PLS: plastic scintillator; NE213: organic liquid scintillator; Div.: divider; DG535: digital delay; nSD: nano second delay; CFD: constant fraction discriminator; 4F1V: 4-fold 1veto coincidence; GG: gate and delay generator; Disc.: discriminator; Scl.: scaler; QDC: charge-to-digital converter; TDC: time-to-digital converter; GbE: giga bit ethernet.

\subsection{Data taking procedure}

In prior to neutron data taking, at first, alignment of the collimators and the target was carefully checked using the LCS photon beam. For collimator alignment, gadolinium orthosilicate sintillator (GSO), rectangular parallel-piped shape with $76 \mathrm{~mm} \times 76 \mathrm{~mm} \times 180 \mathrm{~mm}$ was placed instead of the target. The photon intensity was monitored using the GSO with changing positions and angles of the collimators. The positions and angles were chosen to provide maximum count rate of the LCS photon.

To confirm the target position, a piece of imaging plate was attached on the front face of the target. The beam image of the plate was read by a scanner. The position of image was exactly at the center of the target cylinder. The beam size at the target was $2.3 \mathrm{~mm}$ in diameter.

The polarization of the laser was confirmed using transmitted laser. The results were that the polarization degree was $99.3 \%$.

After these confirmations, neutron measurements were performed to linear and circular polarized beams. The laser system provides vertical polarized beam. The circular polarized beam was prepared using $1 / 4 \lambda$ plate in front of the laser. The running time of the measurement was 47 hours.

For each beam condition, count rate of PLS was calibrated using the GSO. Figure 4 shows setup for the calibration. The GSO detector was placed on the beam line to count.

Stability of neutron detectors was also checked using photon check sources.

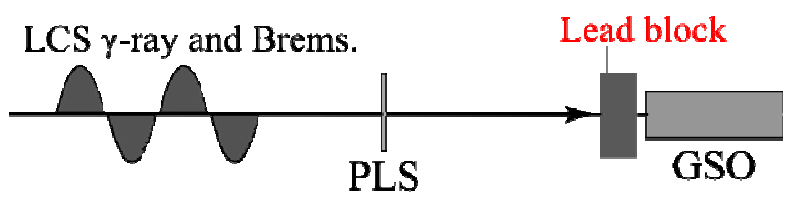

Fig. 4. Experimental geometry used in that the PLS detector is calibrated with the GSO detector.

\section{Data analysis}

\subsection{Number of LCS photon}

To obtain the number of photons, the count rates of the PLS was calibrated with the GSO. Figure 4 shows experimental setup for this calibration. As shown in this figure, a lead block with various thicknesses was placed in front of the GSO to multi-gate pileup of pulses.

Figure 5 (a) and (b) show pulse height spectra of the GSO and the PLS for laser on and off condition. The GSO pulse height spectrum is for $5 \mathrm{~cm}$ in thickness lead. From the data for laser off, contribution from bremsstrahlung can be seen since the electron bunches interact in the storage ring. Therefore, the laser off events was subtracted from laser on to obtain LCS photon contribution. After this subtraction, the counts of the PLS and the GSO were summed for the pulse height channels from $100 \mathrm{ch}$ to $200 \mathrm{ch}$ and from $100 \mathrm{ch}$ to 8192 ch, respectively.

Figure 6 shows thickness of the lead block versus a ratio of count for the GSO to that for the PLS. The data were fitted by an exponential function. As a result, an attenuation coefficient is 0.62 in this measurement. Figure 7 shows the counts for the PLS versus that for the GSO. The counts of the GSO were evaluated with the attenuation coefficient. The data were fitted by a linear function, and the conversion factor from the counts for the PLS detector to the counts for the GSO detector was $111.02 \pm 1.15$. 

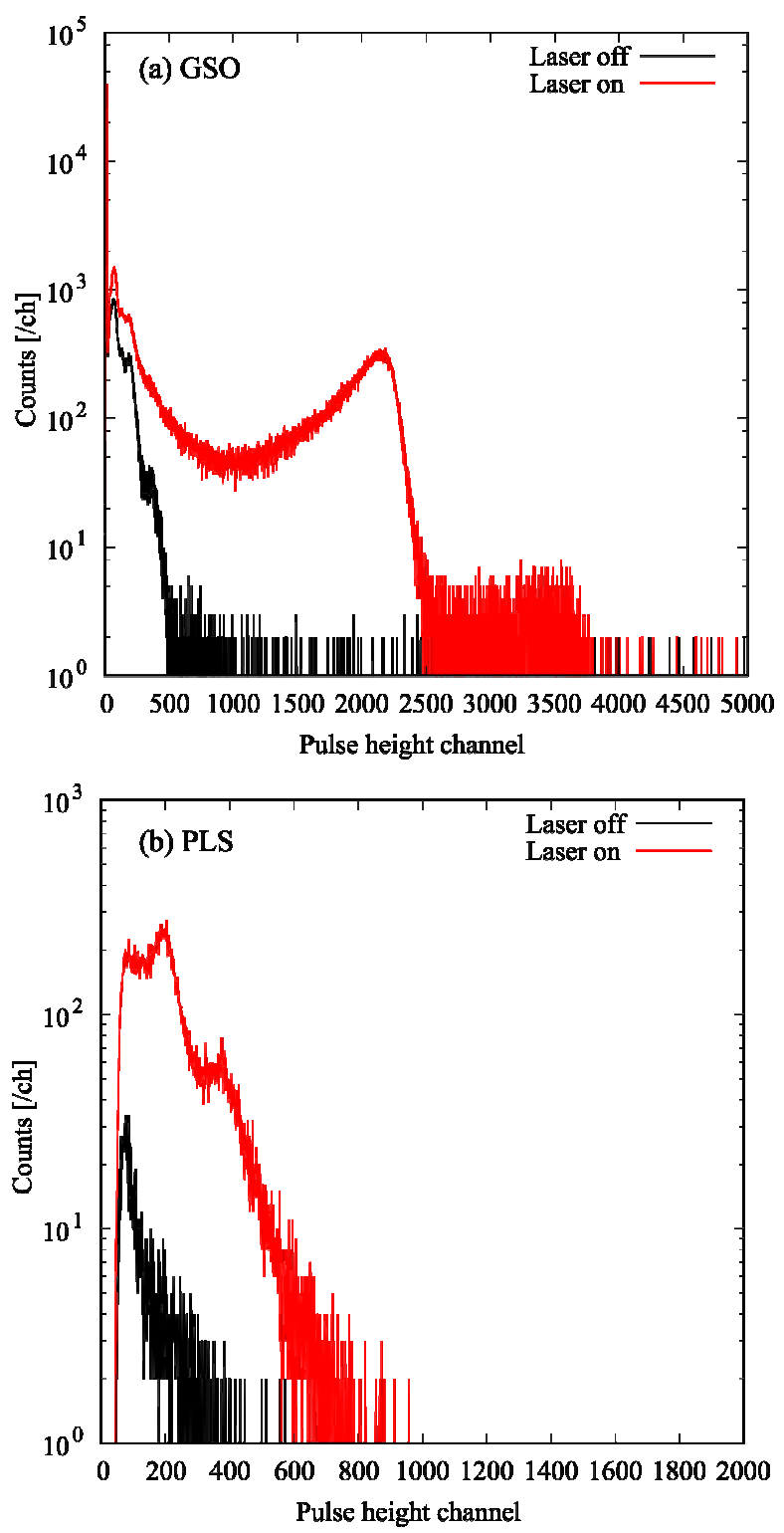

Fig. 5. Pulse height spectra of (a) the PLS detector and (b) the GSO detector for a lead block of $5 \mathrm{~cm}$ thickness. Red line: laser on; black line: laser off.

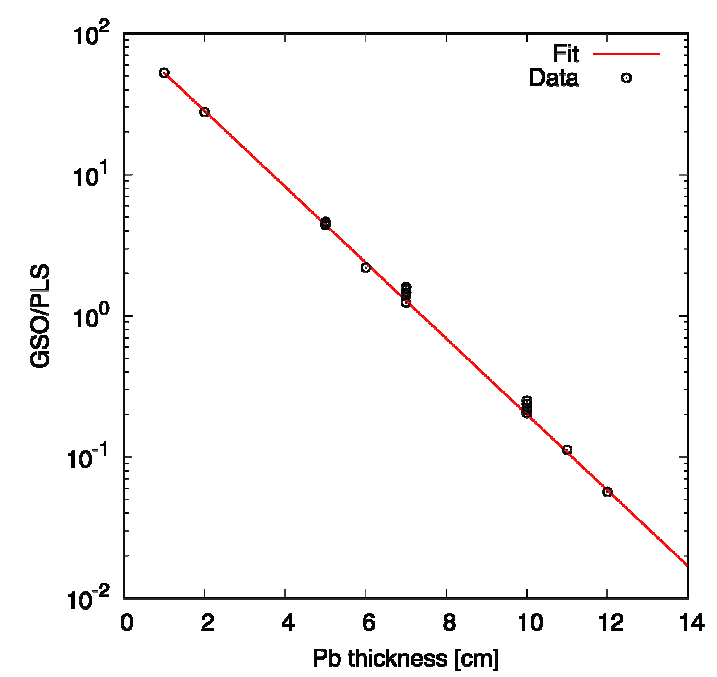

Fig. 6. Thickness of the lead block versus ratio of the count for the GSO to those for the PLS. Red line is the fit by the exponential function.

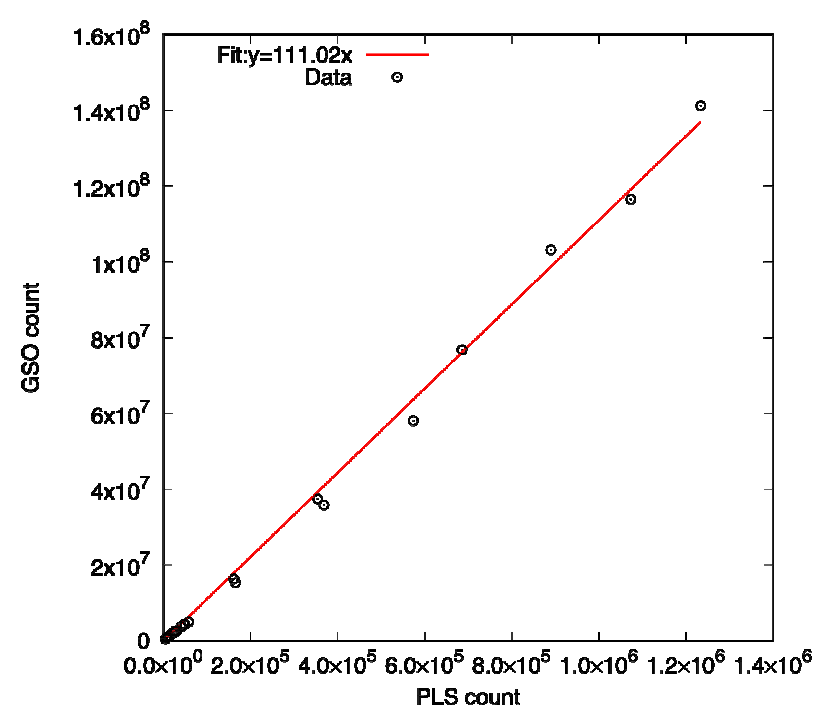

Fig. 7. Counts for the PLS detector versus counts for the GSO detector. The red line is the fit by the linear function.

\subsection{Neutron and gamma separation}

Figure 8 shows a 2D plot for the total component of the pulse-height versus the slow component of the same. The events having data greater than $0.25 \mathrm{MeVee}$ (equivalent to a half of the Cs bias) were chosen. Clear neutron-gamma separation was obtained for three detectors.

Figure 9 shows the TOF spectrum of raw data and data with neutron-gamma separation. The neutron component and the peak of the $\gamma$-ray events are clearly separated. From the FWHM of the $\gamma$-ray peak, we determined that the time resolution was $0.81 \mathrm{~ns}$.

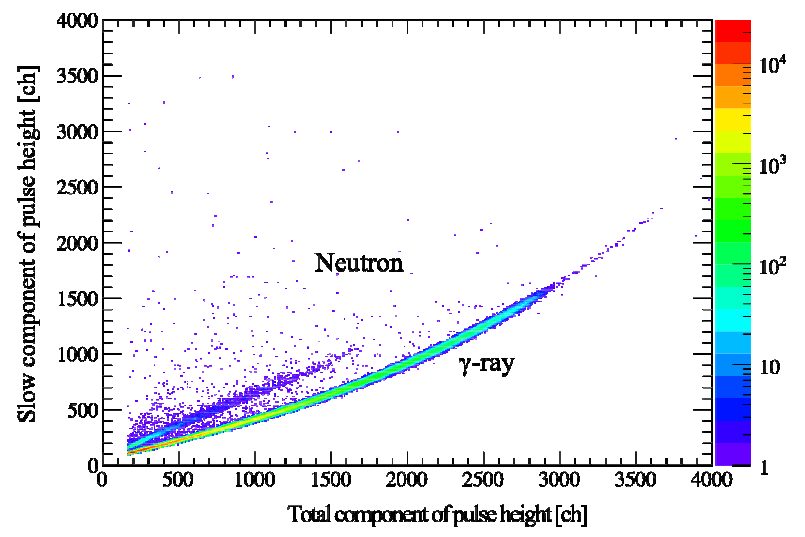

Fig. 8. 2D plot for a total component of the pulse height versus a slow component of the same for the NE1 with the linearly polarized photon. 


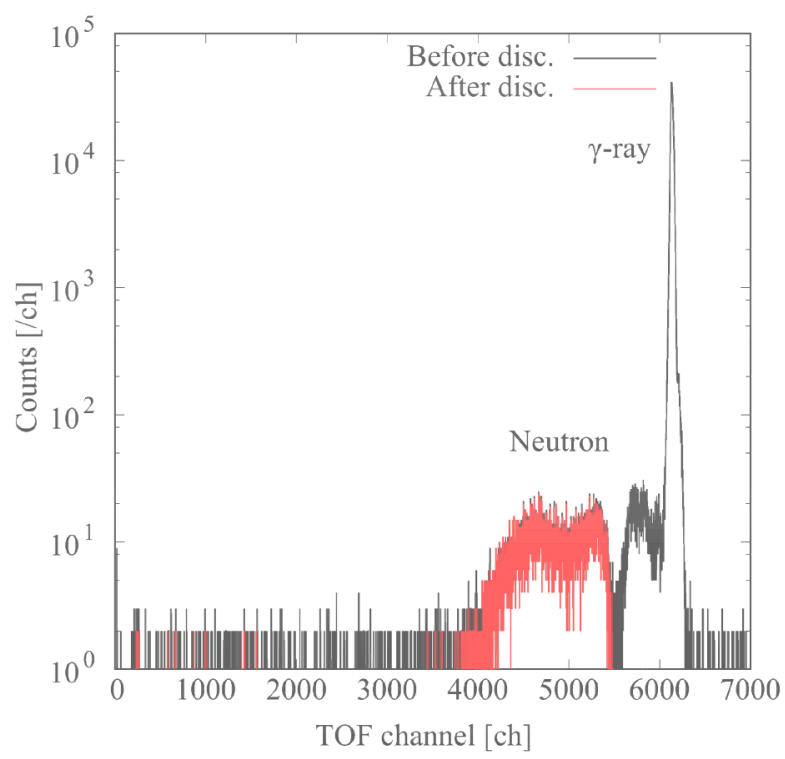

Fig. 9. Time-of-flight spectra for the NE3 detector. Black line: TOF spectrum of raw data; red line: TOF spectrum of neutron event after the pulse-shape discrimination.

\subsection{Neutron detection efficiency}

The efficiency of the NE213 detectors required to transforming the TOF spectrum into the energy spectrum was derived using a SCINFUL-QMD code [8]. Generally, the efficiency of NE213 detectors in low energy region is difficult to estimate purely by calculation because of detector-dependent pulse-height resolution. Thus, the efficiencies of the NE213 detectors for the energies $\leq 3 \mathrm{MeV}$ were derived experimentally using a ${ }^{252} \mathrm{Cf}$ neutron source, the spectrum of which is well known. To estimate the efficiencies of the NE213 detectors, the neutron spectra of the spontaneous fission of ${ }^{252} \mathrm{Cf}$ were measured using a TOF method, and compared with the evaluated spectrum by the National Bureau of Standards (NBS) [9]. The NBS spectrum $X_{\mathrm{Cf}}(E)$ is obtained as

$$
X_{\mathrm{Cf}}(E)=0.6672(E)^{\frac{1}{2}} \exp \left(-\frac{E}{1.42}\right) \mu(E)
$$

where the values of $\mu(E)$ are listed in Table 2 [10]. The relative efficiency was deduced by dividing the observed energy distribution of ${ }^{252} \mathrm{Cf}$ fission neutrons $Y(E)$ by $X_{\mathrm{Cf}}(E)$ :

$$
\varepsilon(E)=C \frac{Y(E)}{X_{\mathrm{Cf}}(E)},
$$

where $C$ is the normalization factor. Figure 10 shows the detector efficiencies determined by ${ }^{252} \mathrm{Cf}$ measurement and the SCINFUL-QMD code for a $0.25 \mathrm{MeVee}$ bias. To estimate the efficiencies by ${ }^{252} \mathrm{Cf}$ measurement for energies $\leq 3 \mathrm{MeV}$, the efficiencies taken by ${ }^{252} \mathrm{Cf}$ measurement were normalized with those determined by the SCINFUL-QMD code between 3 and $5 \mathrm{MeV}$.
Table 2. The values of $\mu(E)$ in Eq (1) [10].

\begin{tabular}{|c|c|}
\hline $\begin{array}{c}\text { Energy interval } \\
{[\mathrm{MeV}]}\end{array}$ & $\mu(E)$ \\
\hline $0-0.25$ & $1+1.20 E-0.237$ \\
\hline $0.25-0.8$ & $1-0.14 E+0.098$ \\
\hline $0.8-1.5$ & $1+0.24 E-0.0332$ \\
\hline $1.5-6.0$ & $1-0.00062 E+0.0037$ \\
\hline $6.0-20$ & $1.0 \exp [-0.03(E-6.0]$ \\
\hline
\end{tabular}

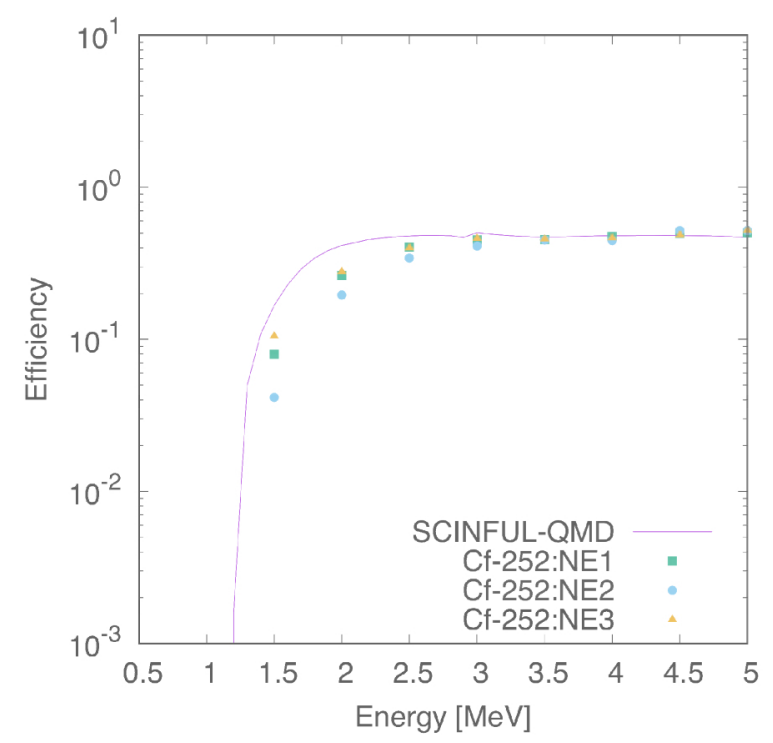

Fig. 10. Detector efficiencies determined by ${ }^{252} \mathrm{Cf}$ measurement and SCINFUL-QMD code for a 0.25 MeVee bias.

\subsection{Uncertainty}

The uncertainties were estimated as shown in Table 3. The uncertainty of the measured number of neutron was evaluated with the statistical uncertainty with each energy. The uncertainty of number of photon was evaluated with determination accuracy of the counts for the GSO to that for the PLS. The uncertainty of solid angle was evaluated with the uncertainty of the detector position. The uncertainty of detector efficiency was the uncertainty of SCINFUL-QMD code. The total uncertainty was estimated with a root mean square of these uncertainties.

Table 3. The values of uncertainties.

\begin{tabular}{|c|c|}
\hline Uncertainty elements & $\begin{array}{c}\text { Uncertainty } \\
{[\%](1 \sigma)}\end{array}$ \\
\hline $\begin{array}{c}\text { Uncertainty of the measured number } \\
\text { of neutron }\end{array}$ & $2-15$ \\
\hline Uncertainty of number of photon & 5 \\
\hline Uncertainty of solid angle & 0.2 \\
\hline Uncertainty of detector efficiency & 5 \\
\hline Total uncertainty & $7-17$ \\
\hline
\end{tabular}

\section{Results and discussion}

\subsection{Energy spectra}

Figure 11 shows the energy spectra of neutron from the ${ }^{197} \mathrm{Au}$ target produced by the photonuclear reaction 
with the circularly and the linearly polarized photons. The spectra were divided with the photon flux and solid angles. At energies $\leq 4 \mathrm{MeV}$, the neutron yield decreased exponentially as the neutron energy increased. Between $4 \mathrm{MeV}$ and $9 \mathrm{MeV}$, the neutron yield was flat.

For the linearly polarized photons, the spectrum, which is took at the same direction of the linear polarization $\left(\phi=90^{\circ}\right)$, is higher than the spectra of the other directions between neutron energies of $4 \mathrm{MeV}$ and $9 \mathrm{MeV}$. This difference was due to the influence of the linear polarization, which was absent for the circularly polarized photons.
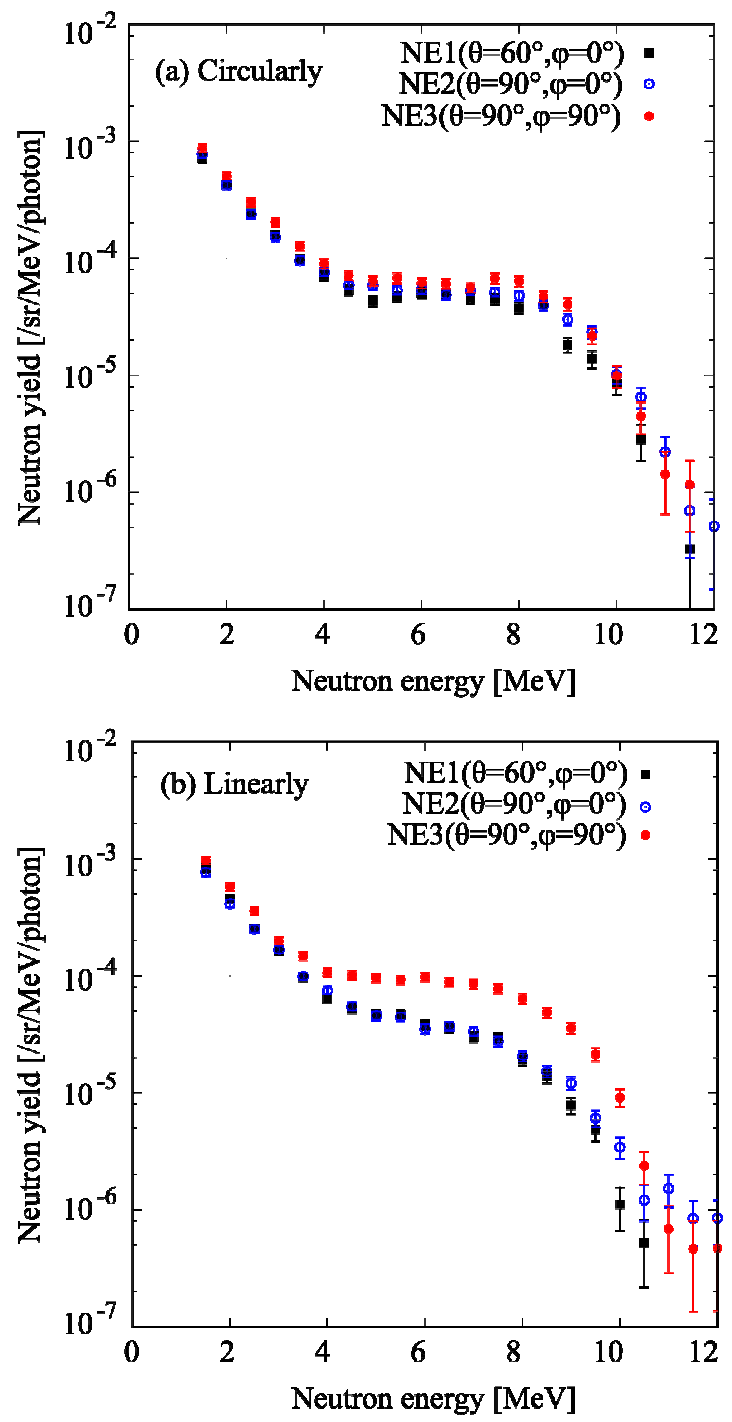

Fig. 11. Neutron energy spectra generated from the ${ }^{197} \mathrm{Au}$ target due to the photonuclear reaction with (a) the circularly and (b) the linearly polarized photons.

\subsection{Comparison with previous data}

Figure 12 shows the ratios of the sum of the NE3 to that of the NE2 as a function of the lower neutron energy. The experimental value produced by Horikawa et al. shows a black point in this figure. The lower energy of the experimental value produced by Horikawa et al. was evaluated from a TOF spectrum in their paper [3]. The ratios in this work reproduce the experimental value produced by Horikawa et al. roughly.

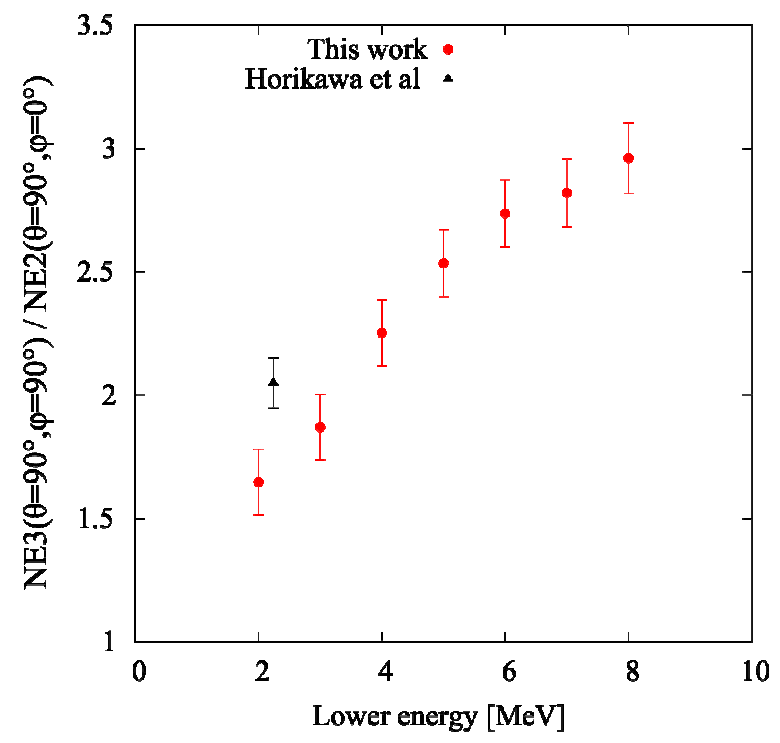

Fig. 12. Ratio of summation for the NE3 to that for the NE2 as a function of lower neutron energy. A black point is the experimental value by Horikawa et al.

\subsection{Energy dependence}

To estimate the energy dependency of ratios of the neutron yield, the ratios of the neutron yield of NE3 ( $\phi=$ $\left.90^{\circ}\right)$ to that of NE2 $\left(\phi=0^{\circ}\right)$ were calculated, as shown in Figure 13 . For the circularly polarized photons, the ratio was approximately constant. For the linearly polarized photons, the ratio increased with the neutron energy, up to approximately three times its initial value.

To estimate the energy dependency between the linearly polarized photon and the circularly polarized photon, ratios of the neutron yields of the linearly polarized photon to those of the circularly polarized photon were calculated, as shown in Figure 14. For the NE3, the ratio was greater than one for the entire neutron energy. For NE1 and NE2, both of which were set perpendicular to the direction of the linear polarization, the ratios decreased as the neutron energy increased. 


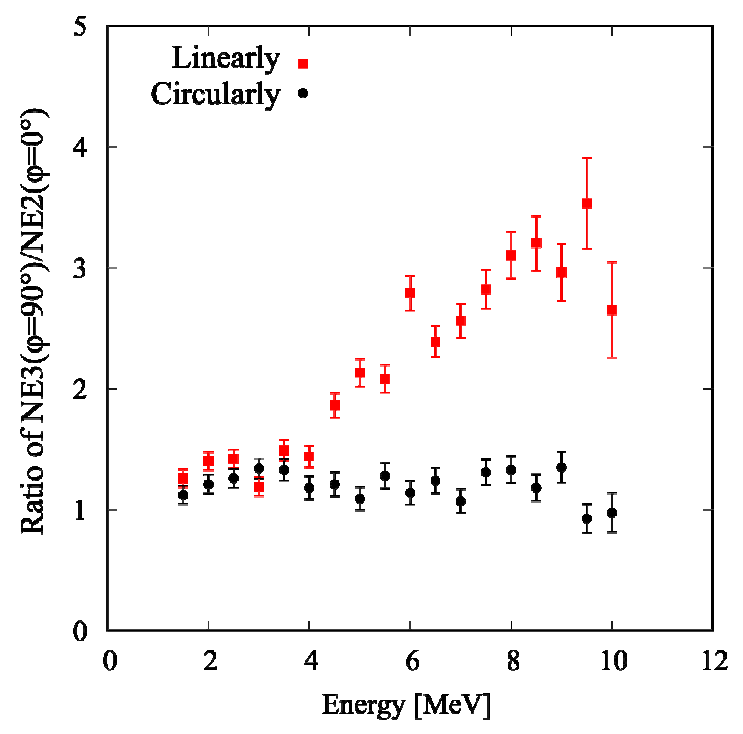

Fig. 13. Ratio of the neutron yield of NE3 to those of NE2.

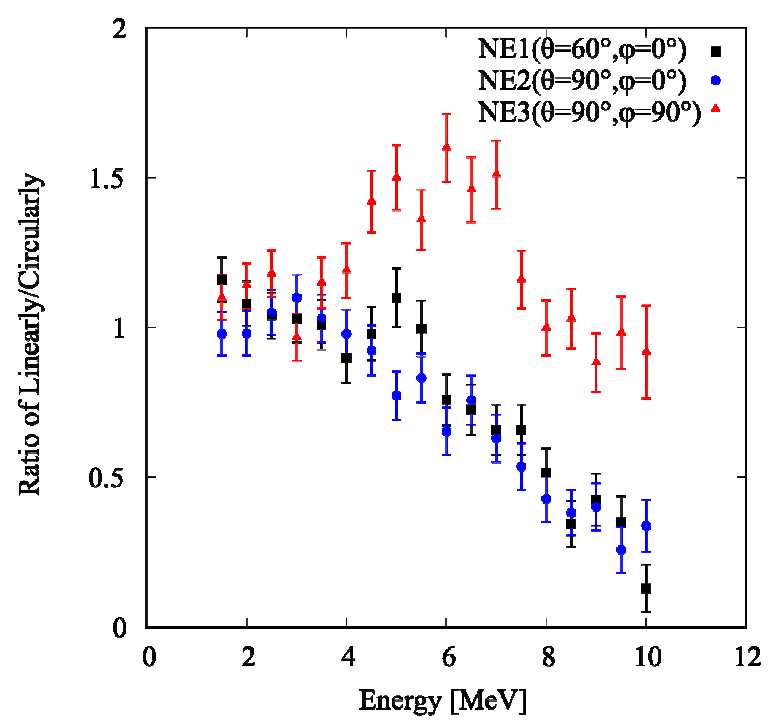

Fig. 14. Ratio of the neutron yield of the linearly polarized photons to that of the circularly polarized photons.

\section{Conclusions}

To estimate the influence of a photonuclear reaction with polarized photons, the neutron spectra generated from a ${ }^{197} \mathrm{Au}$ target by the photonuclear reaction caused by $16.95 \mathrm{MeV}$ polarized LCS $\gamma$-ray beams were measured using a TOF method at NewSUBARU-BL01. The neutron yield that was measured at the same direction as the linear polarization was higher than the yields in the other directions. This difference in the neutron yield increased with the neutron energy, up to a factor of approximately three. The ratios of the sum of the NE3 to that of the NE2 as a function of the lower neutron energy were compared with the experimental value produced by Horikawa et al. The ratios in this work reproduce the experimental value produced by Horikawa et al. roughly. To estimate the energy dependency between the linearly polarized photon and the circularly polarized photon, ratios of the neutron yields of the linearly polarized photon to those of the circularly polarized photon were calculated. In a direction perpendicular to that of the linear polarization, the ratio of the neutron yield decreased as the neutron energy increased.

\section{References}

1. International Linear Collider Technical Design Report, https://www.linearcollider.org/ILC/Publications/Tec hnical-Design-Report

2. V.V. Varlamov, B.S. Ishkhanov, "Study of Consistency Between $(\gamma, \mathrm{xn}),[\gamma, \mathrm{n})+(\gamma, \mathrm{np})]$ and $(\gamma$, 2n) Reaction Cross Sections Using Data Systematics", IAEA, Vienna, Austria, INDC (CCP)433 (2002)

3. K. Horikawa et al., Phys. Lett. B 737, 109 (2014)

4. A. Agodi, Nuovo Cimento, 5, 21, (1) (1957)

5. S. Miyamoto et al., Radiat. Meas. 41, S179 (2007)

6. Y. Asano et al., Prog. Nucl. Sci. Tech. 4, 254 (2014)

7. S. Miyamoto, "NewSUBARU Laser Compton Gamma-ray Source and Study on Photo-nuclear Reaction", Japan Forum of Nuclear Astrophysics, (2009.7.27-7.29)

8. D. Satoh, et al., Nucl. Sci. Tech. 2, 657 (2002)

9. T. Win, "Experimental Studies on Fast NeutronInduced Fission Spectra for ${ }^{237} \mathrm{~Np}$ and ${ }^{233} \mathrm{U} ", \mathrm{PhD}$ thesis, Tohoku University (1999).

10. W. A. G. Sauerwein et al., "Neutron Capture Therapy", Springer Verlag Berlin Heidelberg, (2012). 